\title{
基于横向供应与维修比例的民机备件配置 优化技术研究
}

冯蕴雯, 刘雨昌, 薛小锋, 路成

(西北工业大学 航空学院, 陕西 西安 710072)

\begin{abstract}
摘 要: 针对考虑横向供应的多级库存配置问题,将维修比例这一实际因素引入库存配置模型中,提 出了一种考虑横向供应及维修比例的民机备件多级库存配置方法。首先, 阐述了考虑横向供应及维 修比例下的多级库存配置模型的原理, 并以机队可用度和备件满足率为约束条件, 建立了相应的以库 存系统总成本为优化目标的数学模型; 其次,运用边际分析法求解考虑横向供应及维修比例条件下的 备件多级库存配置; 最后, 以某航空公司波音 737 客机的部附件作为研究对象, 在考虑横向供应及维 修比例的条件下对其进行多级库存配置研究。算例结果得到了最优的备件库存配置数量, 并且得到 最低的库存系统成本为 120.5 万美元, 机队可用度为 0.9909 。相比不考虑横向供应及维修比例的传 统配置模型以及只考虑维修比例的库存配置模型, 所提出的模型使库存成本显著减少, 可为民机备件 的多级库存优化配置提供理论支撑和方法指导。
\end{abstract}

\section{关 键 词: 维修比例; 横向供应;多级库存配置;边际分析法; 民机备件 \\ 中图分类号:V267 \\ 文献标志码: A \\ 文章编号 : 1000-2758(2018)06-1059-10}

备件是飞机维修保障的物质基础, 合理的备件 供应保障策略对飞机持续安全运营有着重要的影 响。备件库存不足将会影响飞机的飞行可靠性与签 派可靠度, 库存过多又会带来高昂的库存保管费用, 因此开展备件的库存优化配置研究有着重要的实际 意义。在民用航空领域, 传统的维修保障模式是由 航站直接向基地申请备件补给, 没有考虑航站之间 发生横向供应。而在实际工程中, 某一航站发生备 件短缺, 为了保障飞机的正常运营, 直接从相邻航站 进行备件补给这种情况会经常发生, 这种情况被称 为横向供应模式, 并且横向供应模式会比传统保障 模式节约更多的时间与成本。但在目前横向供应问 题的研究中, 尚没有考虑维修比例 (即维修站点中 能够修复的故障件数量占送修此站点的故障件总数 的比例) 这一实际因素的影响。在实际中, 若故障 件在基地不能被修复则会进行报废处理, 并购置新 的备件, 因此若不全面考虑维修比例这一因素的影 响将使库存配置结果与实际需求存在差异。为解决
这一问题本文开展考虑横向供应及维修比例的备件 多级库存配置方法研究以确定更经济、有效的库存 配置方案, 为民机备件的多级库存优化配置提供 指导。

国内外学者对备件多级库存配置问题进行了较 多研究, 并且取得了一定成果。如 Sherbrooke 考虑 维修保障组织的多级结构, 提出了 METRIC (multiechelon technique for recoverable item control) 理论用 于多级库存配置研究 ${ }^{[1]}$; 孙蕾等建立了基于 METRIC 理论的多级库存优化模型, 并运用边际分 析法对民用飞机关键部件的库存进行优化 ${ }^{[2]}$; Wayne 等以美国空军保障模式为背景对多级保障组 织的优化配置模型进行了研究 ${ }^{[3]}$ 。以上研究虽然 在多级库存配置模型中涉及了维修比例这一因素, 但仅考虑了航站的维修比例, 而基地仍具有无限修 理能力, 没有将维修比例这一因素考虑到基地维修 过程中, 与实际工程不相符致使库存配置结果与实 际需求存在差异。而 Lee 对单个备件建立了考虑横 
向供应的 2 级库存配置模型, 并基于 METRIC 用近 似估计的方法推导了备件需求可以直接满足、可以 通过横向供应满足以及备件发生短缺的概率 ${ }^{[4]}$; Axsäter 对相似的库存配置模型进行分析, 着重考虑 需求量建模的准确性, 并利用生灭过程推导了在任 一库存水平下的稳态概率 ${ }^{[5]}$; Wong 等结合某航空 公司运营数据, 以系统可用度为约束对横向供应下 的多级库存配置模型进行研究, 以减少航空公司维 修保障成本以及系统的备件延误时间 ${ }^{[6]}$; Jung 等针 对可修件的 2 级可修问题, 以备件满足率为约束对 横向供应下 2 级库存配置系统进行研究 ${ }^{[7]}$; 刘任洋 等在部队现有保障模式背景下, 以装备可用度作为 保障效能约束指标建立了考虑横向供应的 3 级库存 配置模型, 并利用边际分析法进行库存的优化配 置 $^{[8]}$ 。上述研究对考虑横向供应影响下的库存配 置问题进行了分析, 论证了横向供应模式的有效性 与经济性, 但没有考虑维修比例这一实际因素的影 响, 使得库存配置结果与实际需求存在差异。此外, 薛陶等考虑实际中存在报废率的情况, 以最小化维 修成本为目标构建了 2 级单层的 $K / N$ 冷备份圥余 系统可修件优化模型 ${ }^{[9]}$; 冯蕴雯等结合准更新理 论、排队论和 VARI-METRIC 模型提出了考虑不完 全维修的民机可修件多级库存规划方法 ${ }^{[10]}$ 。虽然 上述工作针对备件的多级库存配置问题建立了不同 的库存配置优化模型, 但是在具体应用中仍然存在 一些不足: (1) 文献 [1-3] 没有全面考虑维修比例这 一因素, 虽然引人航站的维修比例, 但仍然认为基地 具有无限的修理能力, 没有考虑到基地存在维修比 例会产生报废并需要重新购置备件的实际情况, 使 得模型的库存配置结果与实际不符; (2) 文献 [4-8, 11-14]针对横向供应模式下的备件多级库存优化配 置进行研究, 验证了横向供应模型的经济有效, 但没 有在模型中考虑维修比例这一实际因素的影响, 使 得库存配置结果与实际需求存在着差异。

为了解决上述问题, 本文针对民机备件的多级 库存配置问题, 结合民机维修保障模式, 提出了一种 考虑维修比例及横向供应的备件多级库存配置研究 方法。首先, 在考虑横向供应库存配置模型中引人 维修比例这一实际因素, 建立备件多级库存配置模 型, 并以备件满足率与机队可用度为约束条件, 以库 存系统总成本为优化目标建立数学模型; 其次, 运用 边际分析法对备件在多级维修保障模式下的库存配 置进行优化; 最后, 结合某航空公司运营数据, 以波
音 737 客机典型部附件作为研究对象进行实例验 证, 并与传统库存配置模型以及只考虑维修比例的 非横向供应库存配置模型进行对比, 验证了本文模 型的有效性与可行性。

\section{1 问题描述与假设}

目前,航空公司多采用 2 级的维修保障体系, 即 航线级与基地级。传统的备件维修保障模式是由航 站直接向基地申请进行备件补给, 但对民机系统而 言,各航站间的距离相比离基地的距离会近得多,如 果某一航站发生备件短缺, 直接从相邻航站进行备 件的补给,将会比传统保障模式节约更多的时间与 成本, 这种由同级之间航站进行备件补给的方式就 是横向供应。此外, 在民机的 2 级维修保障体系中, 航站与基地都具有一定的维修比例, 即故障件中能 够被修复的数量占故障件总数的比例。但以往的研 究中对这一因素的分析不够全面,虽然引人航站的 维修比例，但都假设基地的修理能力为无限大。这 种假设与实际情况不符, 由于维修比例这一因素的 存在, 基地不能实现对故障件的完全修理,会存在着 一定的报废量,这会对备件的多级库存配置产生影 响。为此,本文针对民机备件的航线可更换单元 ( line replaceable units, LRU), 开展考虑横向供应与 维修比例的 2 级单层库存配置研究。

民机的库存配置系统由航线级仓库、相应的航 站维修站点、基地仓库和相应的基地维修站点组成。 民机库存配置系统如图 1 所示, 基地仓库和相应的 修理站点数量为 $m$, 航站仓库和相应的修理点数量 为 $n$, 系统中 LRU 数量为 $p$, 且备件在航线级会发生 横向供应。库存配置系统示意图如 1 所示。

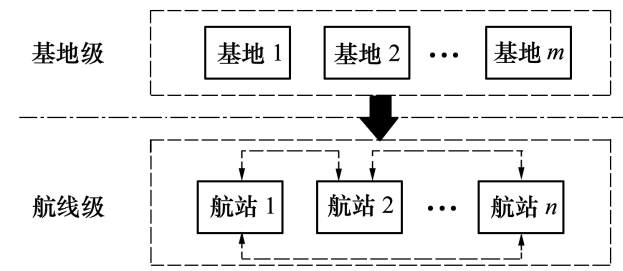

图 1 横向供应模式下 2 级单层库存配置系统示意图

在多级修理模式下,通常按照航站与基地的距 离, 将航站划分为几个不同的共享组, 且只在共享组 内允许横向供应的发生, 共享组内一般设有 2 个或 3 个航站。当飞机在某航站发生 LRU 故障时,如果 
该 LRU 在航站仓库有备件, 则更换故障件, 直接满 足需求; 如果航站仓库中没有备件, 则向临近的航站 提出横向供应申请, 并由提供备件的航站向基地仓 库提出补货申请; 当共享组内的航站中都没有库存 时,则认为出现短缺, 此时向基地仓库申请备件补 给, 飞机停飞待件。拆换下来的故障件先在航站维 修站点进行修理,航站有一定的维修比例, 修理完成 则送往航站仓库; 若不能修理, 则将故障件送往基地 进行修理。基地也存在一定的维修能力, 修理完成 的备件送往基地仓库; 若基地也不能修理, 则将故障 件报废, 订购新的备件补充到基地仓库。当备件在 后方基地修理完成后, 若没有横向供应发生, 则送回 原航站; 若存在横向供应情况, 则送到有需求的航站 仓库(横向供应中提供备件的航站)。考虑横向供 应与维修比例的多级备件库存配置流程图如图 2 所示。

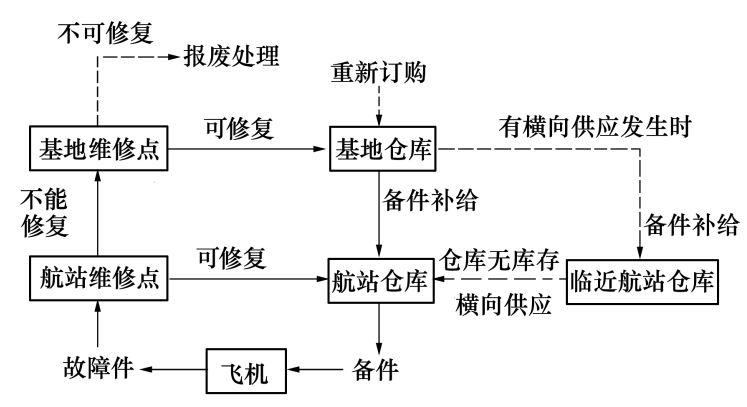

图 2 考虑维修比例及横向供应的两级 单层备件库存配置流程图

在上述的 2 级单层的横向供应库存配置过程 中, 仓库对 LRU 采用减少 1 件补充 1 件的 $(S-1, S)$ 库存补货策略, 并且满足经典库存平衡公式 ${ }^{[2]}$ :

$$
s=s_{O H}+s_{D I}-s_{B O}
$$

式中, $s$ 为仓库的初始库存量, 且 $s$ 为一定值始终不 变; $s_{O H}$ 为某一时刻仓库的库存量; $s_{D I}$ 为某一时刻仓 库的供应渠道数, 即正在维修的 LRU 数量以及在运 输途中的 LRU 数量; $s_{B O}$ 为某一时刻仓库 LRU 的短 缺数量。当有 $\mathrm{LRU}$ 故障件进行维修时, $s_{D I}$ 将增加 1 个, 若仓库有库存, 则 $s_{O H}$ 减少 1 个, 否则 $s_{B O}$ 增加 1 个; 当故障件维修完成时, $s_{D I}$ 减少 1 个, $s_{O H}$ 增加 1 个 或者 $s_{B O}$ 减少 1 个。

为了简化建模过程以及提高模型的工程适用 性, 可以对备件库存优化模型合理地做出如下假设:

1) 所有 LRU 均为可修件, 且 LRU 的故障服从 泊松分布,不同故障件的维修时间相互独立;
2) 认为飞机型号均相同, 且 LRU 的重要程度 相同,一旦发生短缺将导致飞机停飞;

$3)$ 每个航站的库存控制采用 $(S-1, S)$ 库存补 货策略;

4) 航线级的备件可以实现共享, 当某航站发生 需求且没有库存时, 允许在共享组内通过横向转运 从其他航站获得所需备件,但不可以跨组进行;

$5)$ 组内存在 2 个以上的库存点时, 需要明确转 运点的选择原则, 即先向距离近的航站申请, 只有在 近距离的航站没有存货时, 才向稍远距离的航站 申请;

6) 发生横向供应时, 可以立马从相邻航站获得 备件,不考虑延误时间;

7) 认为基地级与航线级, 以及航线级不同航站 之间的运输过程中, 无故障发生。

\section{2 考虑横向供应的 2 级备件库存模型}

\section{1 基地与航站的年平均需求量与供应渠道数}

航空公司的飞机在运营过程中 LRU 的故障随 时都有可能发生, 某 LRU 的年平均需求量等于其 1 年内的平均更换量。每项 LRU 的年平均需求量可 根据(2)式得到

$$
\lambda_{i}=\frac{F \cdot Q_{i} \cdot N}{M_{i}}
$$

式中, $\lambda_{i}$ 为每项 $\mathrm{LRU}$ 的年平均需求量, $F$ 为飞机年 飞行时间, $Q_{i}$ 为每项 LRU 的单机安装数量, $N$ 为机 队规模, $M_{i}$ 为每项 LRU 的平均拆换间隔时间 (MTBUR)。

飞机在一条航线上可能会经过多个航站,所以 航站对某 LRU 的需求量可以根据航程计算得到。 航站 $j$ 中每项 LRU 的正常年平均需求量为

$$
\lambda_{i}^{j}=\frac{D_{j}}{\sum_{j=1}^{n} D_{j}} \lambda_{i}
$$

式中, $\lambda_{i}^{j}$ 为航站 $j$ 中每项 LRU 的正常年平均需求量, $D_{j}$ 为航站 $j$ 与前一航站之间的航程, $\sum_{j=1}^{n} D_{j}$ 为航线总 航程。

航站与基地都具有一定的维修比例, 在航站修 理不了的故障件将送往基地进行修理。所以基地某 $\mathrm{LRU}$ 的年平均需求量为所有在基地修理完成的 $\mathrm{LRU}$ 故障件之和。基地中每项 $\mathrm{LRU}$ 的年平均需求 
量为

$$
\lambda_{i}^{0}=\rho_{i}^{0} \cdot \sum_{j=1}^{n} \lambda_{i}^{j} \cdot\left(1-\rho_{i}^{j}\right)
$$

式中, $\lambda_{i}^{0}$ 为基地中每项 $\mathrm{LRU}$ 的年平均需求量, $\rho_{i}^{0}$ 为 基地中每项 LRU 的维修比例, $\rho_{i}^{j}$ 为航站 $j$ 中每项 LRU 的维修比例。

因为基地维修比例这一实际因素的存在, 基地 修理能力不为无限大, 需要对在基地不能修理的故 障件进行报废处理。所以基地的年平均报废量, 即 为在基地不能修理的 LRU 故障件之和。所以基地 中每项 $\mathrm{LRU}$ 的年平均报废量 $\lambda_{i}^{s}$ 为

$$
\lambda_{i}^{s}=\left(1-\rho_{i}^{0}\right) \cdot \sum_{j=1}^{n} \lambda_{i}^{j} \cdot\left(1-\rho_{i}^{j}\right)
$$

根据模型假设 LRU 的失效服从独立的泊松分 布, 由 Palm 定理可得, 基地中每项 LRU 的供应渠道 数为

$$
\mu_{i}^{0}=\lambda_{i}^{0} \cdot \nu_{i}^{0}
$$

式中, $\mu_{i}^{0}$ 为基地中每项 $\mathrm{LRU}$ 的供应渠道数, $\nu_{i}^{0}$ 为每 项 LRU 在基地的年平均修理时间。

由报废产生的供应渠道数为

$$
\mu_{i}^{s}=\lambda_{i}^{s} \cdot \nu_{i}^{s}
$$

式中, $\mu_{i}^{s}$ 为每项 $\mathrm{LRU}$ 报废产生的供应渠道数, $T_{i}^{s}$ 为 每项 LRU 的重新订购时间。

航站仓库补充备件的平均延误时间由 4 部分组 成: (1)故障件在航站进行修理的时间; (2) 由运输导致 的延误时间; (3)因基地仓库短缺导致的延误时间 ; (4) 因报废产生短缺导致的延误时间。航站 $j$ 中每项 LRU 的平均补货延误时间 $1 / \gamma_{i}^{j}$ 为

$$
\frac{1}{\gamma_{i}^{j}}=\nu_{i}^{j} \cdot \rho_{i}^{j}+\left(1-\rho_{i}^{j}\right) \cdot\left(T_{i}^{j}+D_{i}^{0}+D_{i}^{s}\right)
$$

式中, $\nu_{i}^{j}$ 为每项 LRU 在航站 $j$ 的年平均修理时间, $T_{i}^{j}$ 为第 $i$ 项 LRU 由基地运往航站 $j$ 的运输时间。根据 Little 公式 ${ }^{[8]}$, 因基地仓库短缺导致的延误时间 $D_{i}^{0}=$ $\frac{E_{B O}(s)_{i}^{0}}{\lambda_{i}^{0}}$; 因报废产生短缺导致的延误时间 $D_{i}^{s}=$ $\frac{E_{B O}(s)_{i}^{s}}{\lambda_{i}^{s}} 。$

由 Palm 定理, 航站中每项 LRU 的供应渠道 数为

$$
\mu_{i}^{j}=\frac{\lambda_{i}^{j}}{\gamma_{i}^{j}}
$$

\section{2 横向供应下航线的需求量}

当考虑横向供应时,航站之间会发生备件的横 向转运,航站的需求量会根据备件库存的有无发生 变化。为分析航线的需求量变化引人 3 个参数, $\alpha_{i}^{j}$ 为航站仓库中有库存, 需求可以直接满足的概率; $\beta_{i}^{j}$ 为航站仓库中无库存, 可以由横向供应满足的概率; $\theta_{i}^{j}$ 为航站发生短缺的概率。

当航站中没有备件库存时, 其需求量等于正常 年平均需求量减去年均由其他航站横向供应来的备 件数。所以航站 $j$ 中第 $i$ 项 LRU 无库存时的需求量 $h_{i}^{j}$ 为

$$
h_{i}^{j}=\lambda_{i}^{j}-\frac{\lambda_{i}^{j} \cdot \beta_{i}^{j}}{\left(1-\alpha_{i}^{j}\right)}
$$

当航站中有库存时,其需求量等于正常年平均 需求量加上年均需要转运到其他航站的备件数量。 所以航站 $j$ 中第 $i$ 项 LRU 有库存时的需求量 $g_{i}^{j}$ 为

$$
g_{i}^{j}=\lambda_{i}^{j}+\sum_{l=1}^{J} \frac{\lambda_{i}^{l} \cdot \beta_{i}^{l}}{\alpha_{i}^{j}} \cdot P_{i j}^{l}
$$

式中, $J$ 为共享组内的航站数量, $l$ 为发出横向供应 申请的航站编号, $P_{i j}^{l}$ 为由共享组内航站 $j$ 作为航站 $l$ 的备件供应源的条件概率。当共享组内只有 2 个航 站时, 条件概率 $P_{i j}^{l}$ 为 1 ; 当共享组内有 3 个或 3 个以 上的航站时, 选择横向供应的备件供应源时需要考 虑横向供应的转运成本, 而转运成本与航站间的距 离远近有关, 即先从距离最近的航站进行备件补给, 只有当最近的航站没有备件时再选择其他航站进行 补给, 计算条件概率 $P_{i j}^{l}$ 时需要考虑航站补给的先后 顺序以及临近航站的仓库是否有库存。

在共享组内, 每一个航站的库存系统都可以看 作是一个生灭过程 ${ }^{[5]}$ 。建立航站的库存状态转移 过程如图 3 所示。

图中每一个库存状态对应于一个库存水平, 设 航站 $j$ 中第 $i$ 项 $\mathrm{LRU}$ 的库存水平为 $S_{i}^{j}$, 在这一库存水 平下的稳态概率为 $\pi_{S_{i}^{i}}^{i j}$, 由此可得基地的库存状态 转移方程如下 ${ }^{[6]}$ : 

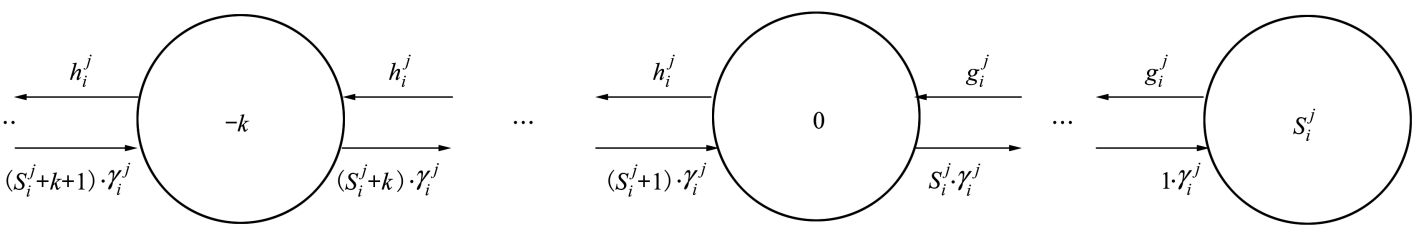

图 3 航站库存状态转移过程图

$\pi_{S_{i}^{i}}^{i j} \cdot g_{i}^{j}=\pi_{S_{i}-1}^{i j} \cdot \gamma_{i}^{j}$

$\pi_{0}^{i j} \cdot\left(h_{i}^{j}+S_{i}^{j} \cdot \gamma_{i}^{j}\right)=\pi_{1}^{i j} \cdot g_{i}^{j}+\pi_{-1}^{i j} \cdot\left(S_{i}^{j}+1\right) \gamma_{i}^{j}$

$\pi_{S_{i}-k}^{i j} \cdot\left(g_{i}^{j}+k \gamma_{i}^{j}\right)=\pi_{S_{i}-k+1}^{i j} \cdot g_{i}^{j}+\pi_{S_{i}-k-1}^{i j} \cdot(k+1) \gamma_{i}^{j}$ $k=1,2, \cdots, S_{i}^{j}$

$\pi_{S_{i-k}}^{i j} \cdot\left(h_{i}^{j}+k \cdot \gamma_{i}^{j}\right)=\pi_{S_{i}-k+i}^{i j} h_{i}^{j}+\pi_{S_{i-k-i}}^{i j}(k+1) \gamma_{i}^{j}$

$$
k=S_{i}^{j}+1, S_{i}^{j}+2, \cdots, \infty
$$

可以得到在某一库存水平下的稳态概率为

$$
\pi_{S_{i}^{i j}}^{i j}=\left\{\begin{array}{l}
\pi_{0}^{i j} \cdot \frac{S_{i}^{j} !}{k !} \cdot\left(\frac{\gamma_{i}^{j}}{g_{i}^{j}}\right)^{S_{i}-k} k=0,1,2, \cdots, S_{i}^{j}-1 \\
\pi_{0}^{i j} \cdot \frac{S_{i}^{j} !}{k !} \cdot\left(\frac{\gamma_{i}^{j}}{h_{i}^{j}}\right)^{-\left(S_{i}-k\right)} k=S_{i}^{j}, S_{i}^{j}+1, \cdots
\end{array}\right.
$$

由各库存水平下的稳态概率之和为 1 , 可得

$$
\frac{1}{\pi_{0}^{i j}}=\sum_{k=0}^{S_{i}-1} \frac{S_{i}^{j} !}{k !} \cdot\left(\frac{\gamma_{i}^{j}}{g_{i}^{j}}\right)^{\left(S_{i}^{i}-k\right)}+\sum_{k=S_{i}^{j}}^{\infty} \frac{S_{i}^{j} !}{k !} \cdot\left(\frac{\gamma_{i}^{j}}{h_{i}^{j}}\right)^{-\left(S_{i}^{i}-k\right)}
$$

由此可得到

$$
\left\{\begin{array}{c}
\alpha_{i}^{j}=\sum_{k=1}^{S_{i}} \pi_{k}^{i j} \\
\theta_{i}^{j}=\prod_{j=1}^{J} \sum_{k=0}^{-\infty} \pi_{k}^{i j} \\
\beta_{i}^{j}=1-\alpha_{i}^{j}-\theta_{i}^{j}
\end{array}\right.
$$

\section{3 基地与航线的期望短缺量}

基地中每项 $\mathrm{LRU}$ 的期望短缺数计算公式为

$$
E_{B O}(s)_{i}^{0}=\sum_{k=s+1}^{+\infty}(k-s) \cdot \frac{\left(\mu_{i}^{0}\right)^{k} \mathrm{e}^{-\mu_{i}^{0}}}{k !}
$$

基地中每项 $\mathrm{LRU}$ 由报废产生的期望短缺数为

$$
E_{B O}(s)_{i}^{s}=\sum_{k=s+1}^{+\infty}(k-s) \cdot \frac{\left(\mu_{i}^{s}\right)^{k} \mathrm{e}^{-\mu_{i}^{s}}}{k !}
$$

航站中每项 LRU 的期望短缺数为

$$
E_{B O}(s)_{i}^{j}=\sum_{k=0}^{-\infty}(-k) \cdot \pi_{k}^{i j}
$$

\section{4 数学模型}

研究民机库存优化配置模型的目的就是希望在 满足机队可用度和备件满足率的前提下, 使库存系 统的总成本达到最低。因此建立以库存系统总成本 为优化目标, 以机队可用度和备件满足率为约束条 件的 2 级横向供应库存配置优化模型

$$
\left\{\begin{array}{c}
\min C \\
\text { s.t. } A \geqslant A_{\min } \\
P_{i} \geqslant P_{i(\min )}
\end{array}\right.
$$

式中, $C$ 为库存系统总成本, 系统的机队可用度 $A=$ $\prod_{j=1}^{n} \prod_{i=1}^{p} A_{i}^{j}, A_{\text {min }}$ 为机队可用度的规定最小值, 系统每 项 LRU 的备件满足率 $P_{i}=\prod_{j=1}^{n} P_{i}^{j}, P_{i(\min )}$ 为每项 $\mathrm{LRU}$ 的备件满足率的最小值。

航站中每项 LRU 的机队可用度为 ${ }^{[13]}$

$$
A_{i}^{j}=\left(1-\frac{E_{B O}(s)_{i}^{j}}{N \times Q_{i}}\right)^{Q_{i}}
$$

航站中每项 LRU 的备件满足率为

$$
P_{i}^{j}=\sum_{k=0}^{S}\left(\frac{\left(\mu_{i}^{j}\right)^{k}}{k !} e^{-\mu_{i}^{j}}\right)
$$

\section{3 考虑横向供应与维修比例的备件库 存配置模型优化流程}

本文库存配置模型的优化算法选用边际分析 法。虽然边际分析法的求解精度没有遗传算法的精 度高, 但是边际分析法在求解民机备件库存配置这 一类复杂系统问题上具有更高的效率, 是一种国内 外普遍采用的方法。 


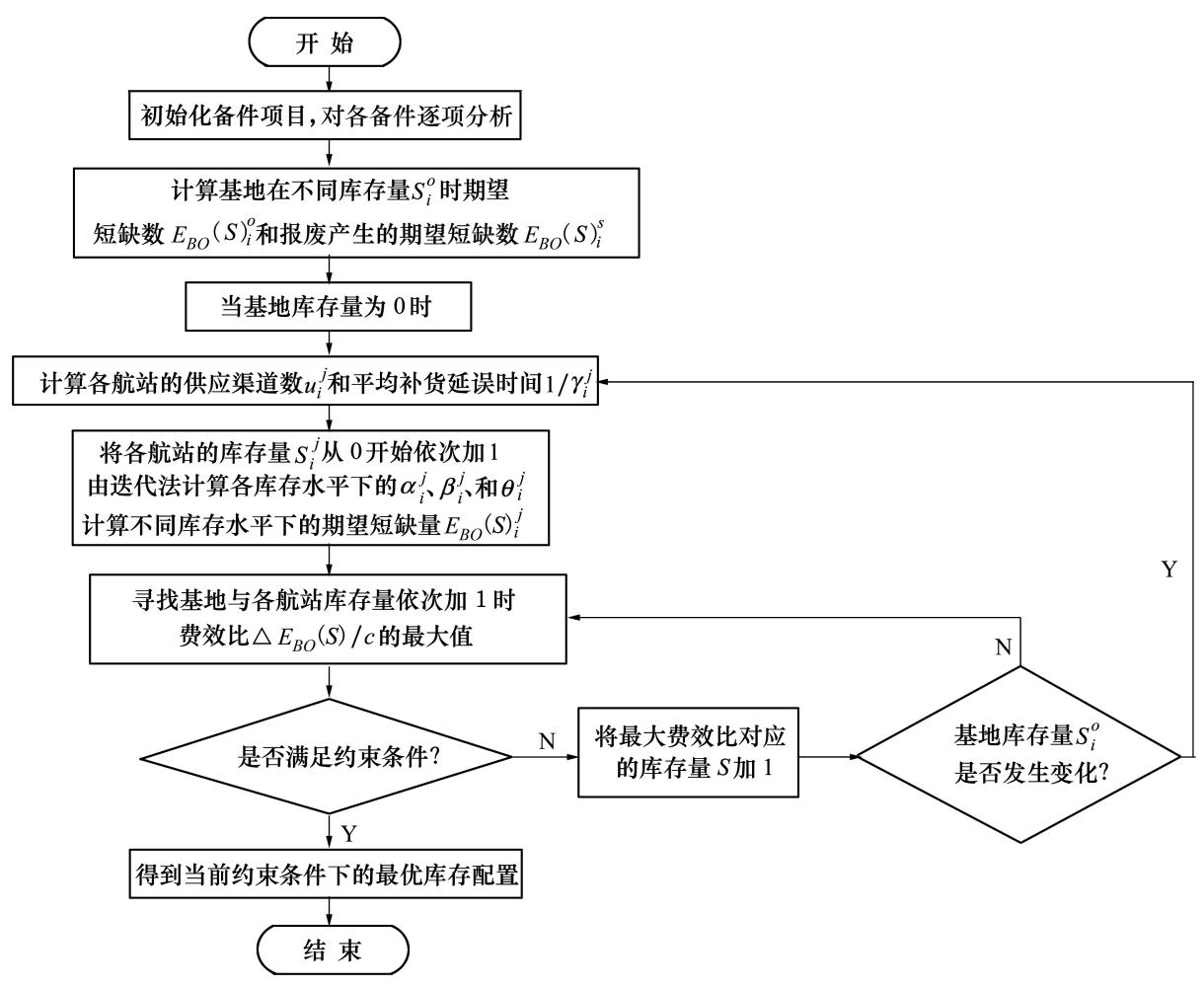

图 4 边际分析法库存配置流程图

库存配置模型的优化流程为:

1) 首先根据公式 (2) (3) 计算各航站对每个 LRU 的年平均需求量, 根据航站的年均需求量按照 (4) （5) 式计算基地的年平均需求量和年平均报 废量;

2) 初始化基地的库存量, 使基地的备件数量 为 0 。

3) 按照公式(6) (7) 计算基地以及因报废产 生的供应渠道数,由（18） (19) 式计算基地和因报 废产生的期望短缺数 $E_{B O}$ 。

4) 根据 (8) 式计算各个航站在库存量为 0 时的 平均补货延误时间,利用迭代法计算各个航站的 $\alpha_{i}^{j}$, $\beta_{i}^{j}$ 和 $\theta_{i}^{j}$, 并计算各航站的期望短缺数。

具体的迭代方法为: 先给定 $\alpha_{i}^{j}$ 和 $\theta_{i}^{j}$ 一个初始 值, 根据 (10) (11) 式计算航站有库存时的需求 量 $g_{i}^{j}$ 和无库存时的需求量 $h_{i}^{j}$, 代人库存状态转移方 程 (16) (17) 可以计算出不同库存水平状态下的 稳态概率。由 (18) 式可得一组新的 $\alpha_{i}^{j}$ 和 $\theta_{i}^{j}$ 的值, 与 初始值进行比较, 若不小于误差水平 $e$, 则令新的 $\alpha_{i}^{j}$ 和 $\theta_{i}^{i}$ 作为初始值重新进行迭代; 若小于误差水平, 停 止迭代。

5) 由 (19) (21) 式计算基地与各航站在不同
库存量下的期望短缺数, 并建立费效比矩阵。

$$
\left\{\begin{array}{l}
\Delta E_{B O}(s)_{i}^{0}=\left(E_{B O}(s)_{i}^{0}-E_{B O}(s+1)_{i}^{0}\right) / c_{i} \\
\Delta E_{B O}(s)_{i}^{s}=\left(E_{B O}(s)_{i}^{s}-E_{B O}(s+1)_{i}^{s}\right) / c_{i} \\
\Delta E_{B O}(s)_{i}^{j}=\left(E_{B O}(s)_{i}^{j}-E_{B O}(s+1)_{i}^{j}\right) / c_{i}
\end{array}\right.
$$

6) 寻找费效比最大的位置, 给费效比最大的位 置库存量加一, 直到机队可用度和备件保障率满足 约束条件。

7) 当基地的库存量发生变化时,航站的平均补 货延误时间也会发生变化,所以返回第 4)、5)步更 新航站的费效比矩阵。

\section{4 算例分析}

本文以某航空公司波音 737 客机的 5 个起落架 LRU 作为分析对象, 且服从上文中的模型假设条 件,机队由 10 架相同型号的 737 客机组成,年平均 飞行小时为 $3000 \mathrm{~h}$, 机队可用度最小值设为 0.99 。 库存系统由 1 个基地和 5 个航站组成, 其中航站 1 , 2 组成共享组 1 , 航站 $3,4,5$ 组成共享组 2 , 且航站 间距离 $D_{34}<D_{35}<D_{45}$ 。库存系统示意图如下。各项 LRU 参数如表 1 所示。 


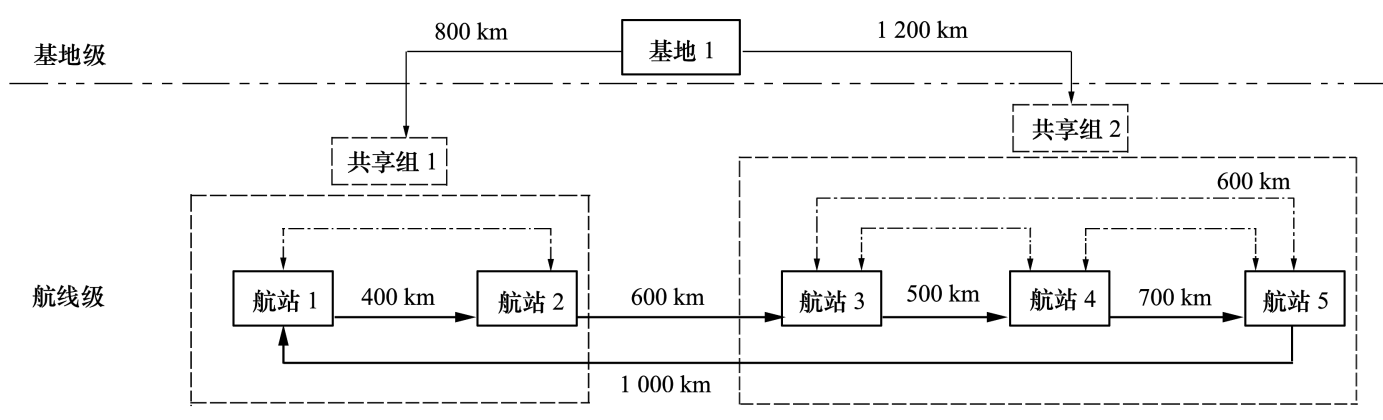

图 5 起落架 LRU 库存配置系统示意图

表 1 起落架 $\mathrm{LRU}$ 参数表

\begin{tabular}{cccccccccc}
\hline LRU 编号 & $M_{i} / \mathrm{h}$ & $C_{i} /$ 美元 & $Q_{i}$ & $\rho_{i}^{0}$ & $\rho_{i}^{j}$ & $\nu_{i}$ & $\nu_{i}$ & $T_{i}^{\mathrm{s}}$ & $P_{i(\min )}$ \\
\hline 1 & 1500 & $2.7 \times 10^{4}$ & 2.0 & 0.6 & 0.4 & 0.12 & 0.10 & 0.17 & 0.97 \\
2 & 1000 & $3.3 \times 10^{4}$ & 2.0 & 0.7 & 0.5 & 0.16 & 0.14 & 0.18 & 0.96 \\
3 & 1500 & $2.9 \times 10^{4}$ & 1.0 & 0.8 & 0.6 & 0.14 & 0.13 & 0.17 & 0.97 \\
4 & 300 & $1.8 \times 10^{4}$ & 3.0 & 0.6 & 0.4 & 0.15 & 0.12 & 0.17 & 0.96 \\
5 & 300 & $0.5 \times 10^{4}$ & 2.0 & 0.7 & 0.5 & 0.17 & 0.15 & 0.20 & 0.95 \\
\hline
\end{tabular}

根据上述各航站的运营信息以及各项 LRU 的 基本参数, 由公式 $(2) \sim(9)$ 计算基地以及各航站的 年平均需求量与供应渠道数, 再根据 $(10) \sim(18)$ 式 按照迭代法计算各航站的在不同库存水平下的稳态 概率, 由 (19) (23) 式计算得到基地与各航站的期 望短缺数、备件保障率以及机队可用度。由此可以 建立以库存系统总成本为优化目标, 以机队可用度 与备件保障率为约束条件的民机起落架 LRU 在横 向供应情况下的两级单层备件库存优化模型。通过 边际分析法配置各项 LRU 在基地与各航站的库存 数量, 库存配置情况如表 2 所示。

表 2 考虑横向供应以及维修比例的起落架 LRU 库存配置

\begin{tabular}{ccccccc}
\hline $\begin{array}{c}\text { 库存 } \\
\text { 系统 }\end{array}$ & LRU1 & LRU2 & LRU3 & LRU4 & LRU5 & $\begin{array}{c}C_{i} / \\
\text { 美元 }\end{array}$ \\
\hline 基地 & 6 & 5 & 4 & 7 & 7 & $60.4 \times 10^{4}$ \\
航站 1 & 1 & 1 & 1 & 2 & 2 & $13.5 \times 10^{4}$ \\
航站 2 & 1 & 1 & 1 & 2 & 1 & $13 \times 10^{4}$ \\
航站 3 & 1 & 1 & 1 & 1 & 1 & $11.2 \times 10^{4}$ \\
航站 4 & 1 & 1 & 1 & 1 & 1 & $11.2 \times 10^{4}$ \\
航站 5 & 1 & 1 & 1 & 1 & 1 & $11.2 \times 10^{4}$ \\
\hline
\end{tabular}

由表 2 可知, 当系统中 LRU 库存总数为 57 件
时, 机队可用度达到 0.9909 , 此时的库存配置系统 的总成本为 120.5 万美元。

为了验证考虑横向供应与维修比例的库存配置 模型的可行性与有效性, 根据上述航站运营信息与 表 1 中各项 LRU 的参数, 分别在考虑维修比例但不 允许发生横向转运以及既不考虑维修比例也不允许 发生横向供应的传统配置模型 2 种情况下, 利用边 际分析法进行备件的库存配置, 系统库存配置情况 如表 3 、表 4 所示。

\section{表 3 考虑维修比例的非横向供应起落架 LRU 库存配置}

\begin{tabular}{ccccccc}
\hline $\begin{array}{c}\text { 库存 } \\
\text { 系统 }\end{array}$ & LRU1 & LRU2 & LRU3 & LRU4 & LRU5 & $\begin{array}{c}C_{i} / \\
\text { 美元 }\end{array}$ \\
\hline 基地 & 7 & 7 & 5 & 8 & 7 & $74.4 \times 10^{4}$ \\
航站 1 & 2 & 2 & 2 & 2 & 3 & $22.9 \times 10^{4}$ \\
航站 2 & 2 & 2 & 2 & 2 & 2 & $22.4 \times 10^{4}$ \\
航站 3 & 2 & 2 & 2 & 2 & 3 & $22.9 \times 10^{4}$ \\
航站 4 & 2 & 2 & 2 & 2 & 2 & $22.4 \times 10^{4}$ \\
航站 5 & 2 & 2 & 2 & 2 & 3 & $22.9 \times 10^{4}$ \\
\hline
\end{tabular}

由表 3 可知, 在不允许发生横向转运时, 系统中 LRU 库存总数需要达到 87 件时, 机队可用度达到 0.9901 , 此时的库存配置系统的总成本为 187.9 万 
美元。

表 4 不考虑横向供应与维修比例的起落架 LRU 库存配置

\begin{tabular}{ccccccc}
\hline $\begin{array}{c}\text { 库存 } \\
\text { 系统 }\end{array}$ & LRU1 & LRU2 & LRU3 & LRU4 & LRU5 & $\begin{array}{c}C_{i} \text { ' } \\
\text { 美元 }\end{array}$ \\
\hline 基地 & 5 & 5 & 3 & 5 & 5 & $50.2 \times 10^{4}$ \\
航站 1 & 2 & 2 & 2 & 2 & 3 & $22.9 \times 10^{4}$ \\
航站 2 & 2 & 2 & 2 & 2 & 2 & $22.4 \times 10^{4}$ \\
航站 3 & 2 & 2 & 2 & 1 & 3 & $21.1 \times 10^{4}$ \\
航站 4 & 2 & 2 & 2 & 2 & 2 & $22.4 \times 10^{4}$ \\
航站 5 & 2 & 2 & 2 & 2 & 3 & $22.9 \times 10^{4}$ \\
\hline
\end{tabular}

由表 4 可知, 在不考虑横向供应与维修比例的 传统配置模型中, 系统 LRU 的库存总数达到 76 件 时, 机队可用度达到 0.9902 , 此时的库存配置系统 的总成本为 161.9 万美元。

通过采用边际分析法对上述 3 种情况下的起落 架 LRU 库存配置进行研究, 可以得到 3 种情况分别 对应的机队可用度与系统库存成本的关系曲线如图 6 所示。

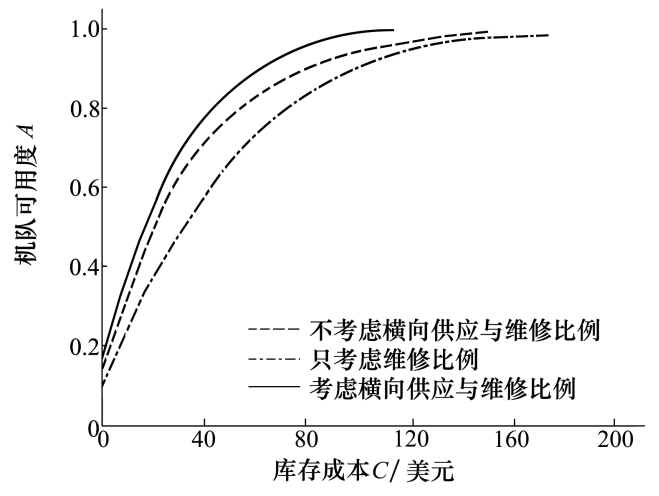

图 6 起落架 $\mathrm{LRU}$ 库存配置系统示意图

由图 6 可以看出, 在满足约束条件的情况下, 考 虑横向供应及维修比例的库存配置模型与只考虑维 修比例的库存配置模型以及不考虑横向供应及维修 比例的库存配置模型相比, 其库存系统总成本分别 减少了 $25.6 \%$ 与 $35.9 \%$ 。这是由于允许横向供应发 生时, 各航站间可以直接进行备件的横向补给, 各航 站只需维持较低的库存数量就可以通过横向供应满 足备件需求, 因此其库存系统总成本相较于其他 2 种情况显著降低。由图还可以看出在满足约束条件 的情况下本文所提出模型的机队可用度高于其他 2 种情况, 且在相同库存成本的条件下, 本文所提出模
型的机队可用度最大。通过对比表 2 、表 3 的库存 配置情况可以看出, 同时考虑 2 级维修比例的情况 下, 当允许横向供应发生时, 因为航站与基地的备件 需求降低, 航站的库存配置数量明显下降, 基地的库 存配置数量也有所降低; 通过对比表 3、表 4 可以看 出各航站的库存配置情况基本相同, 但当考虑维修 比例这一因素的影响时, 由于基地的维修比例不为 无限大, 在基地中存在着一定的报废量, 需要重新购 置备件, 因此考虑维修比例情况下的基地库存配置 数量明显增加; 对比表 2 、表 4 还可以看出考虑横向 供应与维修比例情况下的系统库存配置总数与各航 站的库存配置数量都明显小于传统的库存配置模 型。可以发现横向供应模式对成本的节约效果非常 明显, 可以为航空公司降低大量库存成本, 在此基础 上考虑维修比例的影响, 虽然使基地的库存配置数 量上升,但更符合实际工程的需求。

\section{5 结 论}

本文针对横向供应下的多级库存配置问题,在 库存配置模型中引入维修比例这一实际因素, 提出 了一种考虑维修比例及横向供应的民机备件多级库 存配置研究方法, 并以波音 737 客机起落架 LRU 作 为研究对象进行实例验证, 得到结论如下:

1) 以减少库存系统总费用, 提高机队可用度为 目标, 结合民机维修保障模式, 在横向供应下的库存 配置问题中引人维修比例这一实际工程因素, 建立 了考虑维修比例及横向供应的备件多级库存配置优 化模型。

2) 在考虑 2 级维修比例的情况下, 以某航空公 司波音 737 客机起落架 LRU 作为研究对象, 建立了 以库存配置费用总成本为优化目标, 以系统的机队 可用度与备件保障率为约束条件的横向供应库存配 置模型。通过分析计算, 可以得到基地与各航站的 备件配置数量, 并且当机队可用度达到 0.9909 , 系 统的库存配置总成本最低为 120.5 万美元。

3) 通过与只考虑维修比例的非横向供应配置 模型, 以及不考虑横向供应及维修比例的库存配置 模型进行对比可以发现, 所提出的模型使库存成本 得到显著减少, 并使机队可用度达到最高。验证了 本文提出模型的有效性, 证明了在库存配置问题中 同时考虑横向供应及维修比例的影响更加符合工程 中的实际情况, 为民机备件的多级库存优化配置提 
供理论支撑和方法指导。

\section{参考文献:}

[1] Sherbrooke C C. METRIC-A Multi-Echelon Technique for Recoverable Item Control[ J]. Operations Research, 1968, 16( 1): 122-141

[2] 孙蕾, 左洪福. 基于 METRIC 的民机初始备件数量确定及配置模型 [J]. 南京航空航天大学学报, 2013, 24(23): 3200-3204

Sun Lei, Zuo Hongfu. Optimal Allocation Modeling for Initial Spare Parts of Civil Aircraft Based on METRIC[ J]. Journal of Nanjing University of Aeronautics \& Astronautics, 2013, 24(23):3200-3204

[3] Wayne L Z, Richard F D, Lee J L. Modeling Diminishing Marginal Returns in a Hierarchical Inventory System of Reparable Spare Parts $[J]$. Annals of Operations Research, 1999, 91:319-337

[4] Lee H L. A Multi-Echelon Inventory Model for Repairable Items with Emergency Lateral Transshipments[J]. Management Science, 1987, 33(10): 1302-1316

[5] Axsäter S. Modeling Emergency Lateral Transshipments in Inventory Systems [J]. Management Science, 1990, 36(11):29-40

[6] Wong H, Van Houtum G J, Cattrysse D, et al. Multi-Item Spare Parts Systems with Lateral Transshipments and Waiting Time Constraints $[\mathrm{J}]$. European Journal of Operational Research, 2006, 171(3) : 1071-1093

[7] Jung B R, Sun B G, Kim J S, et al. Modeling Lateral Transshipments in Multi-Echelon Repairable-Item Inventory Systems with Finite Repair Channels[J]. Computers \& Operations Research, 2003, 30(9): 1401-1417

[8] 刘任洋, 李庆民, 李华. 基于横向转运策略的可修件三级库存优化模型 $[\mathrm{J}]$. 航空学报, 2014, 35(12):3341-3349

Liu Renyang, Li Qingmin, Li Hua. Optimal Model of Three-Echelon Inventory for Repairable Spare Parts with Lateral Transshipments Strategy $[\mathrm{J}]$. Acta Aeronautica et Astronautica Sinica, 2014, 35(12):3341-3349 (in Chinese)

[9] 薛陶, 冯蕴雯, 秦强. 考虑报废的 $\mathrm{K} / \mathrm{N}$ 冷备份穴余系统可修复备件优化 $[\mathrm{J}]$. 华南理工大学学报, 2014, 42(1) :41-46

Xue Tao, Feng Yunwen, Qin Qiang. Optimization of Repairable Spare Parts for K/N Cold-Standby Redundant System Considering Scraps [J]. Journal of South China University of Technology, 2014, 42(1) :41-46 (in Chinese)

[10] 冯蕴雯, 李永凯, 薛小锋, 等. 考虑不完全维修的民机可修件多级库存规划 [ J ]. 西北工业大学学报, 2017, 35( 5): 827-833

Feng Yunwen, Li Yongkai, Xue Xiaofeng, et al. Multi-Echelon Inventory Allocation under Imperfect Repair for Repairable Spares of Civil Aircraft [J]. Journal of Northwestern Polytechnical University, 2017, 35(5):827-833 (in Chinese)

[11] Tiacci L, Saetta S. Reducing the Mean Supply Delay of Spare Parts Using Lateral Transshipments Policies[J]. International Journal of Production Economics, 2011, 133(1):182-191

[12] Olsson F. Emergency Lateral Transshipments in a Two-Location Inventory System with Positive Transshipment Leadtimes[J]. European Journal of Operational Research, 2015, 242(2): 424-433

[13] 张光宇, 李庆民, 郭璇. 基于横向转运策略的可修备件多点库存建模方法 [J]. 系统工程与电子技术, 2012, 34(7): 1424-1429

Zhang Guangyu, Li Qingmin, Guo Xuan. Modeling for Repairable Spare Parts in Multi-Location Inventory System with Lateral Transshipments $[\mathrm{J}]$. Systems Engineering and Electronics, 2012, 34(7):1424-1429 (in Chinese)

[14] Patriarca R, Costantino F, Gravio G D. Inventory Model for a Multi-Echelon System with Unidirectional Lateral Transshipment [J]. Expert Systems with Applications, 2016, 65: 372-382

[15] 蔡芝明, 金家善, 陈砚桥. 基于关键性的备件库存配置优化模型 $[J]$. 系统工程与电子技术, 2017, 39(8):1765-1773 Cai Zhimeng, Jin Jiashan, Chen Yanqiao. Optimal Inventory Modeling of Spare Parts under the Criticality [J]. Systems Engineering and Electronics, 2017, 39(8): 1765-1773 


\title{
Research on Configuration Optimization of Civil Aircraft Spare Parts with Lateral Transshipments and Maintenance Ratio
}

\author{
Feng Yunwen, Liu Yuchang, Xue Xiaofeng, Lu Cheng \\ (School of Aeronautics, Northwestern Polytechnical University, Xi'an 710072, China)
}

\begin{abstract}
A research method for multi-echelon inventory allocation of civil aircraft spare parts with lateral transshipments and maintenance ratio is proposed in this paper, and the maintenance rate is considered into the inventory model, aiming at the problem of multi-echelon inventory allocation with lateral transshipments for civil aircraft spare parts. Firstly, the principle of the multi-echelon inventory model with lateral transshipments and maintenance ratio is proposed. Then, by considering the fleet availability and the satisfaction ratio as the constraints, a mathematical model is built with the inventory system total cost as the optimization objective. Secondly, the procedure of solving multi-echelon inventory allocation model considering the influence of lateral transshipments and maintenance ratio is discussed based on the marginal analysis method. Finally, some components of Boeing 737 are selected as the research objects, the multi-echelon inventory allocation is investigated by considering lateral transshipments and maintenance ratio. The analysis results in this paper show the best number of multi echelon inventory allocation, the minimum inventory system total cost and the fleet availability are 1.205 million dollars and 0.9909 respectively. Comparing the traditional inventory model without considering lateral transshipments and maintenance ratio, and the model which only considers the maintenance ratio, the results indicate that the model offered in this paper could reduce the total cost of inventory system significantly, and provides theoretical support and method guidance for the accurate allocation of multi echelon inventory optimization for civil aircraft spare parts.
\end{abstract}

Keywords : maintenance ratio ; lateral transshipments; multi-echelon inventory allocation ; marginal analysis method; civil aircraft spare parts 